\title{
In the Search of Electron Correlation Effects in DNA
}

\author{
A. WRÓBEL ${ }^{a}$ AND P. WRÓBEL ${ }^{b}$ \\ ${ }^{a}$ Institute of Physics, Wrocław University of Technology, Wybrzeże Wyspiańskiego 27, 50-370 Wrocław, Poland \\ ${ }^{b}$ Institute for the Low Temperature and Structure Research, P.O. Box 1410, 50-950 Wrocław 2, Poland
}

We discuss the energy scale separation induced in DNA by gaps between molecular orbital states of individual bases and by electron correlations. We also demonstrate how this separation gives rise to effective low energy models of electron transport in DNA.

PACS numbers: 87.14.gk, 75.20.-g, 71.10.Fd

\section{Introduction}

Several interesting attempts have been made by other authors to construct effective models of electron transport in DNA $[1,2]$. We concentrate in our present research on some important aspects which have not been yet discussed in the full extent. They are relevant energy scales and especially their separation induced by correlation effects and by gaps between the energies of highest occupied molecular orbitals (HOMOs) and the energies of lowest unoccupied molecular orbitals (LUMOs). The construction of the effective model is based on the concept of dividing the molecule into "cells" which are separate molecular fragments. Provided that the separation between them is sufficient one can use the notion of orbitals associated with each fragment. In the case of DNA sequences it is natural to choose nucleosides associated with the bases, adenine (A), cytosine $(C)$, guanine $(\mathrm{G})$, and thymine $(\mathrm{T})$, as such quantum cells. It is thus clear that the effective model will have the structure of a ladder which represents the DNA helix. For each segment we restrict the analysis to HOMO and LUMO states since other states are supposed to lie too far from the Fermi energy. Such a conclusion follows both from the experimental data and from the results of earlier analyses aiming at the determination of relevant parameters. Those analyses have been performed in the framework of the valence-bond/Hartree-Fock (VB/HF) approach [3, 4] and in the framework of the local density approach (LDA) [5].

\section{HOMO-LUMO model}

By using the above mentioned insights we start to construct an effective Hamiltonian which consists of a part $H^{(0)}$ determining the energy scales and of a part $H^{(1)}$ which can be treated as a perturbation. $H^{(0)}$ should contain parameters which refer to the values of molecular orbital eigenenergies and to electron correlations. The first type of above mentioned parameters includes the crucial information on the values of the gaps between different eigenenergies. The size of those gaps together with the values of the Coulomb energy contributions from differ- ent configurations set the energy scales for the phenomena related to electron transport in DNA.

It seems that the minimal form of $H^{(0)}$ containing all necessary ingredients is

$$
\begin{aligned}
& H^{(0)}=\sum_{i ; l ; o} \epsilon_{i l o} n_{i l o}+\sum_{i ; l ; o} U_{i l o} n_{i l o \uparrow} n_{i l o \downarrow} \\
& +\sum_{i ; l} U_{i l \mathrm{HL}} n_{i l \mathrm{H}} n_{i l \mathrm{~L}} \\
& +\sum_{\left\{(i, l) \neq\left(i^{\prime}, l^{\prime}\right)\right\}} V_{i j i^{\prime} l^{\prime}}\left(\sum_{o} n_{i l o}-Z\right) \\
& \quad \times\left(\sum_{o^{\prime}} n_{i^{\prime} l^{\prime} o^{\prime}}-Z\right),
\end{aligned}
$$

where $i$ and $i^{\prime}$ label base pairs, $l$ and $l^{\prime}$ label strands, e.g. $l=1,2$, while $o$ and $o^{\prime}$ refer to the type of orbital, e.g. $o=\operatorname{HOMO}(\mathrm{H})$, LUMO $(\mathrm{L})$. In the last term on the right side of (1) the summation is carried over contributions from pairs of orbitals which either belong to different base pairs or to different strands. $U_{i l o}$ refers to the Coulomb interaction between electrons in the same orbital state of the same molecular segment. $U_{i l \mathrm{HL}}$ represents the value of the same potential for electrons also in a single cell but in different orbital states, while $V_{i j i^{\prime} l^{\prime}}$ refers to the contribution from total charges generated in different molecular fragments. $Z=2$ is the net charge of the segment with empty both HOMO and LUMO, and $n_{i l o}=n_{i l o \uparrow}+n_{i l o \downarrow}$. It seems to be worth to emphasize here that the Hamiltonian part (1) describing correlation effects is more general than those used in some earlier analyses which either concentrated exclusively on HOMO states as in [4] or which dealt from the very begin with a single orbital per molecular segment, either LUMO or HOMO [6]. Since we start with a model which incorporates both types of orbitals for each molecular cell, in our approach correlations between HOMO and LUMO electrons simultaneously created in the same segment are taken into account.

The Hamiltonian part $H^{(1)}$ which will be treated as a perturbation is related to electron transfer between nucleosides, since estimates based on the $\mathrm{VB}$ approach $[3,4]$ and on LDA [5] suggest that energy scale involved in those processes is much smaller than energies related to 
gaps between orbital eigenenergies and to the energies of electron correlations. $H^{(1)}$ can be written in the following form:

$$
\begin{aligned}
& H^{(1)}=-\sum_{i ; o ; o^{\prime}} t_{i o o^{\prime}}\left(c_{i 1 o}^{\dagger} c_{i 2 o^{\prime}}+\text { H.c. }\right)-\sum_{i ; o ; o^{\prime}} t_{(i+1) o i o^{\prime}}^{\prime} \\
& \quad \times\left(\mathrm{e}^{\mathrm{i}\left(2 \pi \Phi / \Phi_{0}\right)\left(\cos \theta_{i+1}+\cos \theta_{i}\right)} c_{(i+1) 1 o}^{\dagger} c_{i 1 o^{\prime}}+\text { H.c. }\right) \\
& -\sum_{i ; o ; o^{\prime}} t_{(i+1) o i o^{\prime}}^{\prime \prime}\left(\mathrm{e}^{-\mathrm{i}\left(2 \pi \Phi / \Phi_{0}\right)\left(\cos \theta_{i+1}+\cos \theta_{i}\right)}\right. \\
& \left.\quad \times c_{(i+1) 2 o}^{\dagger} c_{i 2 o^{\prime}}+\text { H.c. }\right) .
\end{aligned}
$$

The first part of (2) represents interstrand hopping inside a single base pair, while the second and the third represent the intrastrand hopping between bases belonging to nearest pairs. Since we are here interested in the effect of electron correlations and in the effect of the energy scale separation, which is induced by them, on the basic phenomenon involving electron transfer that is orbital magnetism, we have supplemented the hopping term with phase factors according to Peierls substitution prescription, $t \rightarrow t \exp \left(\mathrm{i}\left(2 \pi \Phi / \Phi_{0}\right) \int_{i}^{j} \boldsymbol{A}(\boldsymbol{r}) \mathrm{d} \boldsymbol{r}\right)$. The vector defining positions of bases in the pair $i$ is (i $\left.a, \mp \cos \left(\theta_{i}\right) r / 2, \mp \sin \left(\theta_{i}\right) r / 2\right)$, where upper and lower signs refer to the strands 1 and 2 , respectively. $r \approx 1 \mathrm{~nm}$ is the radius of the DNA helix and simultaneously it is also the distance between centers of bases which belong to the same pair, $a=0.34 \mathrm{~nm}$ is the distance between base pairs, while $\theta_{i}$ refers to their twist angle. We use the following gauge, $\boldsymbol{B}=B x \hat{\boldsymbol{y}} . \Phi=B d a$ refers to the flux directed through a hypothetical untwisted plaquette which has been formed by two base pairs and which is perpendicular to the field.

\section{Separation of energy scales}

Both estimations based on VB [3, 4] approach and on LDA [5] indicate that the values of hopping integrals $t$ are not much bigger than $0.1 \mathrm{eV}$, while the gaps between HOMO states and LUMO states and the energies of electron correlations are much bigger than $1 \mathrm{eV}$. Furthermore, the results of the analysis performed in the framework of the LDA scheme suggest that for both base pairs $\mathrm{A}-\mathrm{T}$ and $\mathrm{G}-\mathrm{C}$, orbital eigenenergies of the first base lie much higher than the orbital eigenenergies of the second. If we choose the HOMO eigenenergy of $\mathrm{C}$ as the reference level, the levels of HOMO eigenenergies are located at about $2.2 \mathrm{eV}$ for $\mathrm{G}, 0 \mathrm{eV}$ for $\mathrm{C}, 1.3 \mathrm{eV}$ for $\mathrm{A}$ and 0.2 , for $\mathrm{T}$, while the levels of LUMO eigenenergies are located at about $7.6 \mathrm{eV}$ for $\mathrm{G}, 5.5 \mathrm{eV}$ for $\mathrm{C}, 6.8 \mathrm{eV}$ for $\mathrm{A}$, and $5.6 \mathrm{eV}$ for $\mathrm{T}$. The form of those energy sequences, schematically presented in Fig. 1a for the base $\mathrm{G}-\mathrm{C}$, and the smallness of the hopping integrals $t$ suggest that low energy excitations, such as the example depicted in Fig. 1b, will involve only a single frontier orbital for each base. In the case of bases $\mathrm{G}$ and $\mathrm{A}$ for which HOMO and LUMO levels lie high, HOMO will play the role of the frontier orbital, while in the case of bases $\mathrm{C}$ and $\mathrm{T}$ for which HOMO and LUMO levels lie low LUMO will

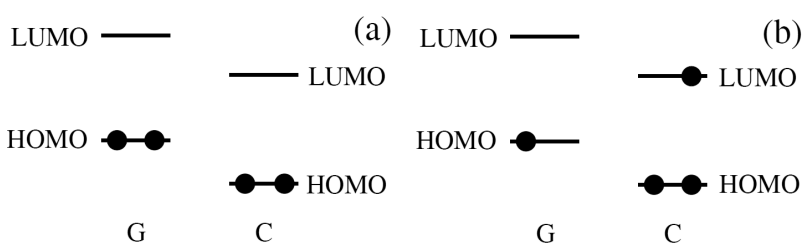

Fig. 1. (a) The level structure of HOMO and LUMO states in the base pair G-C. (b) An example of lowest excitation. The electron transfer occurs between frontier orbitals.

play the role of the frontier orbital. By using this observation we may substantially simplify both segments of the Hamiltonian. The part which sets energy scales is truncated and transformed to

$$
\begin{aligned}
& H^{(0)}=\sum_{i ; l} \epsilon_{b(i l)} n_{i l}+\sum_{i ; l} U_{b(i l)} n_{i l \uparrow} n_{i l \downarrow} \\
& +\sum_{i ; l} U_{b(i l) \mathrm{HL}}\left(2-Z_{b(i l)}\right) n_{i l} \\
& +\sum_{\left\{(i, l) \neq\left(i^{\prime}, l^{\prime}\right)\right\}} V_{i j i^{\prime} l^{\prime}}\left(n_{i l}-Z_{b(i l)}\right) \\
& \quad \times\left(n_{i^{\prime} l^{\prime}}-Z_{b\left(i^{\prime} l^{\prime}\right)}\right),
\end{aligned}
$$

where $\epsilon_{b(i l)}$ is the eigenenergy of the frontier orbital for the base $b(i l)$ located in the base pair $i$ and the strand $l$, $U_{b(i l)}$ is the Coulomb energy of electrons which occupy that orbital, while $U_{b(i l) \mathrm{HL}}$ represents the same potential for electrons which occupy different states, which means both HOMO and LUMO, in a single molecular segment. $Z_{b(i l)}$ refers to the core charge for the state in which the frontier orbital is empty and is $Z_{b(i l)}=2$ if $\mathrm{HOMO}$ is the frontier orbital for the base $b(i l)$ and is $Z_{b(i l)}=0$ if LUMO is the frontier orbital for the base $b(i l)$. That choice guarantees that the third term in (3) representing the interorbital intrabase Coulomb interaction is active only if LUMO is the frontier orbital and HOMO is always full in the low energy sector of the physical space, while it is inactive when HOMO is the frontier orbital and LUMO is empty. The fourth term represents the Coulomb potential of net charges located at different bases. By comparing states depicted in Fig. 1a and b we easily see that Eq. (3) gives the correct value of the energy difference. First three terms produce contributions to that difference, $\epsilon_{\mathrm{C}}-\epsilon_{\mathrm{G}},-U_{\mathrm{G}}, 2 U_{\mathrm{CHL}}$, respectively. Also the fourth term produces the correct value -1 (in the units of $e^{2}$ ) for the product of net charges at bases $\mathrm{G}$ and $\mathrm{C}$. The results of the analysis performed in the framework of the $\mathrm{VB}$ approach suggest that for the parameters $V_{i j i^{\prime} l^{\prime}}$ we may use the values of the bare Coulomb potential

$$
V_{i j i^{\prime} l^{\prime}}=\frac{\mathrm{e}^{2}}{\left|\boldsymbol{R}_{i l}-\boldsymbol{R}_{i^{\prime} l^{\prime}}\right|},
$$

where $\boldsymbol{R}_{i j}$ represents the position vector of the base in the pair $i$, the strand $l$. For the important case of two nearest pairs we use the notation $V_{t}$ for potentials of charges at bases belonging to the same pair, $V_{l}$ for charges at bases belonging to different pairs and the same strand, 
and $V_{d}$ for bases belonging to different pairs and different strands. fied,

The hopping part of the Hamiltonian also gets simpli-

$$
\begin{aligned}
& H^{(1)}=-\sum_{i} t_{p(i)}\left(c_{i 1}^{\dagger} c_{i 2}+\text { H.c. }\right)-\sum_{i ; l} t_{b((i+1) l) b(i l)} \\
& \quad \times\left(\mathrm{e}^{-(-1)^{l} \mathrm{i}\left(2 \pi \Phi / \Phi_{0}\right)\left(\cos \theta_{i+1}+\cos \theta_{i}\right)}\right. \\
& \left.\quad \times c_{(i+1) l}^{\dagger} c_{i l}+\text { H.c. }\right),
\end{aligned}
$$

$t_{p(i)}$ is the integral of hopping between frontier orbitals of different bases inside a single base pair, $p(i)=\mathrm{A}-\mathrm{T}$ or $p(i)=\mathrm{G}-\mathrm{C}$, while $t_{b((i+1) l) b(i l)}$ refers to intrastrand hopping between nearest bases belonging to different pairs, $t_{b((i+1) l) b(i l)}=t_{\mathrm{AC}}, t_{\mathrm{AG}}, \ldots$

\section{Unitary transformation}

We proceed now to present the scheme of unitary transformation based on the standard stationary perturbation theory. The aim of this procedure is to block diagonalize the Hamiltonian in a way which uses the separation of energy scales and decouples the low energy section of the physical space from the high energy section. Energy scales in the analyzed problem are determined by the Hamiltonian part $H^{(0)}$ and $\left|\phi_{n}^{(0)}\right\rangle$ are its low energy eigenstates which are separated from high energy eigenstates $\left|\psi_{n}^{(0)}\right\rangle$ by a gap which is sufficiently big in comparison with the energy scale introduced by the perturbation part $H^{(1)}$ :

$$
\begin{aligned}
& H=H^{(0)}+H^{(1)}, \\
& H^{(0)}\left|\phi_{n}^{(0)}\right\rangle=E_{n}^{(0, \phi)}\left|\phi_{n}^{(0)}\right\rangle, \\
& H^{(0)}\left|\psi_{n}^{(0)}\right\rangle=E_{n}^{(0, \psi)}\left|\psi_{n}^{(0)}\right\rangle .
\end{aligned}
$$

We seek two sets of states,

$$
\begin{aligned}
& \left|\phi_{n}\right\rangle=\left|\phi_{n}^{(0)}\right\rangle+\sum_{m} a_{n m}\left|\phi_{m}^{(0)}\right\rangle+\sum_{m} b_{n m}\left|\psi_{m}^{(0)}\right\rangle, \\
& \left|\psi_{n}\right\rangle=\left|\psi_{n}^{(0)}\right\rangle+\sum_{m} c_{n m}\left|\phi_{m}^{(0)}\right\rangle+\sum_{m} d_{n m}\left|\psi_{m}^{(0)}\right\rangle,
\end{aligned}
$$

which block diagonalize $H$,

$$
\begin{aligned}
& \left\langle\phi_{n} \mid \phi_{n^{\prime}}\right\rangle=\delta_{n, n^{\prime}}, \\
& \left\langle\psi_{n} \mid \psi_{n^{\prime}}\right\rangle=\delta_{n, n^{\prime}}, \\
& \left\langle\phi_{n} \mid \psi_{n^{\prime}}\right\rangle=0, \\
& \left\langle\phi_{n}|H| \psi_{n^{\prime}}\right\rangle=0,
\end{aligned}
$$

and adiabatically evolve from initial states $\left|\phi_{n}^{(0)}\right\rangle$ and $\left|\psi_{n}^{(0)}\right\rangle$, which means that the Taylor expansion of matrix elements $a_{m n}, b_{m n}, c_{m n}$, and $d_{m n}$ starts at the first order with respect to matrix elements of $H^{(1)}$ evaluated for initial states. By postulating that matrices $a_{m n}$ and $d_{m n}$ are Hermitian we can guarantee that the procedure of finding all matrices order by order is unique. Without dwelling upon more details we mention that, in the case of DNA problem which is analyzed by us, the lowest order contribution to the low energy part of the block diagonalized Hamiltonian, restricted to the part relevant to orbital magnetism is

$$
\begin{aligned}
& -\sum_{m, i_{1}, i_{2}, i_{3}, n}\left[\frac{\left|\phi_{m}^{(0)}\right\rangle\left\langle\phi_{m}^{(0)}\left|H^{(1)}\right| \psi_{i_{1}}^{(0)}\right\rangle\left\langle\psi_{i_{1}}^{(0)}\left|H^{(1)}\right| \psi_{i_{2}}^{(0)}\right\rangle}{2\left(E_{i_{1}}^{(0, \psi)}-E_{n}^{(0, \phi)}\right)\left(E_{i_{2}}^{(0, \psi)}-E_{n}^{(0, \phi)}\right)}\right. \\
& \quad \times \frac{\left\langle\psi_{i_{2}}^{(0)}\left|H^{(1)}\right| \psi_{i_{3}}^{(0)}\right\rangle\left\langle\psi_{i_{3}}^{(0)}\left|H^{(1)}\right| \phi_{n}^{(0)}\right\rangle\left\langle\phi_{n}^{(0)}\right|}{\left(E_{i_{3}}^{(0, \psi)}-E_{n}^{(0, \phi)}\right)} \\
& +\frac{\left|\phi_{m}^{(0)}\right\rangle\left\langle\phi_{m}^{(0)}\left|H^{(1)}\right| \psi_{i_{1}}^{(0)}\right\rangle\left\langle\psi_{i_{1}}^{(0)}\left|H^{(1)}\right| \psi_{i_{2}}^{(0)}\right\rangle}{2\left(E_{i_{1}}^{(0, \psi)}-E_{m}^{(0, \phi)}\right)\left(E_{i_{2}}^{(0, \psi)}-E_{m}^{(0, \phi)}\right)} \\
& \left.\quad \times \frac{\left\langle\psi_{i_{2}}^{(0)}\left|H^{(1)}\right| \psi_{i_{3}}^{(0)}\right\rangle \mid\left\langle\psi_{i_{3}}^{(0)}\left|H^{(1)}\right| \phi_{n}^{(0)}\right\rangle\left\langle\phi_{n}^{(0)}\right|}{\left(E_{i_{3}}^{(0, \psi)}-E_{m}^{(0, \phi)}\right)}\right]
\end{aligned}
$$

Since for the problem of orbital magnetism in DNA the low energy sector of the physical space consists of a single state, contributions to (15) can be found by analyzing how the hopping term (5) couples the lowest initial state in which all HOMOs are occupied again with the same state via some excited states. During the physical process associated with that coupling the transferred charge should close a loop, otherwise no contribution to orbital magnetic response would be made. An example of such a process can be seen in Fig. 2a. Labels near positions of

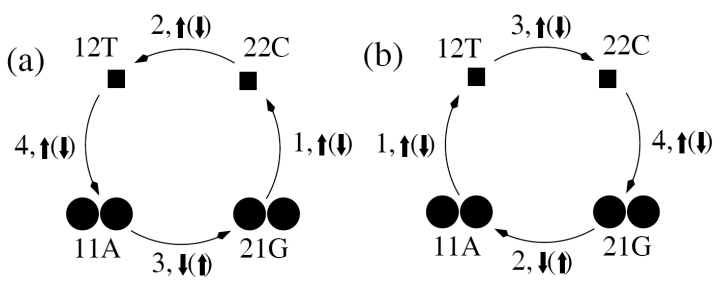

Fig. 2. A process contributing to orbital magnetism of DNA (a) and its time reversed version (b).

bases refer to the pair number, the strand number, and the base type. Filled disks represent bases for which HOMOs are frontier orbitals, while filled squares represent bases for which LUMOs are frontier orbitals. During the first hop an electron with spin up (or alternatively with spin down) is shifted from HOMO at G to LUMO at C, the matrix element $\left(-t_{\mathrm{G}-\mathrm{C}}\right)$ is produced, and the energy difference $\left(\epsilon_{\mathrm{C}}-\epsilon_{\mathrm{G}}-U_{\mathrm{G}}+2 U_{\mathrm{CHL}}-V_{t}\right)$ appears as a factor in the denominators of both terms in (15). In the second step the spin up (down) electron is moved from LUMO at $\mathrm{C}$ to LUMO at $\mathrm{T}$, the related matrix element is $\left(-\mathrm{e}^{\mathrm{i}\left(2 \pi \Phi / \Phi_{0}\right)\left(\cos \theta_{1}+\cos \theta_{2}\right)} t_{\mathrm{TC}}\right)$, and for the energy difference we get $\left(\epsilon_{\mathrm{T}}-\epsilon_{\mathrm{G}}-U_{\mathrm{G}}+2 U_{\mathrm{THL}}-V_{d}\right)$. During the third hop an electron with spin down (up) is shifted from HOMO at $\mathrm{A}$ to $\mathrm{HOMO}$ at $\mathrm{G}$, the matrix element $\left(-\mathrm{e}^{\mathrm{i}\left(2 \pi \Phi / \Phi_{0}\right)\left(\cos \theta_{1}+\cos \theta_{2}\right)} t_{\mathrm{GA}}\right)$ is produced, and the energy difference $\left(\epsilon_{\mathrm{T}}-\epsilon_{\mathrm{A}}-U_{\mathrm{A}}+2 U_{\mathrm{THL}}-V_{t}\right)$ appears in the denominators. The last move of the spin up (down) electron is accompanied by the matrix element $\left(-t_{\mathrm{A}-\mathrm{T}}\right)$. Due to the presence of time reversed process depicted in Fig. 2a the total contribution to the Hamiltonian is Hermitian. 


\section{Conclusions}

The full derivation of the Hamiltonian describing the relevant low energy sector of the physical space for the problem of orbital magnetism in DNA will be presented elsewhere. Nevertheless, already the analysis performed in this report has demonstrated that due to the energy scale separation, the low energy physical space gets much reduced in comparison with the initial space for which basic interactions derived from quantum chemical considerations have been defined. This observation opens promising perspectives for research performed by means of analytical as well as by means of numerical methods.

\section{References}

[1] Modern Methods for Theoretical Physical Chemistry of Biopolymers, Eds. E. Starikov, J.P. Lewis, S. Tanaka, Elsevier Science, Amsterdam 2006.

[2] Charge Migration in DNA: Perspectives from Physics Chemistry, and Biology, Ed. T. Chakraborty, Springer, New York 2007.

[3] G. Brunaud, F. Castet, A. Fritsch, L. Ducasse, Phys. Chem. Chem. Phys. 4, 6072 (2002).

[4] G. Brunaud, F. Castet, A. Fritsch, L. Ducasse, Phys. Chem. Chem. Phys. 5, 2104 (2003).

[5] H. Mehrez, M.P. Anantram, Phys. Rev. B 71, 115405 (2005).

[6] V. Apalkov, T. Chakraborty, Phys. Rev. B 78, 104424 (2008). 